\title{
Growth of Carbon Nanotubes by Pyrolysis of Composite Film of Poly (Vinyl Alcohol) and Modified Fly Ash
}

\author{
Dilip C. D. Nath*, Veena Sahajwalla \\ Centre for Sustainable Materials Research and Technology, School of Materials Science and Engineering, The University of New South \\ Wales, Sydney, Australia. \\ Email: *dilip.nath@unsw.edu.au
}

Received November $16^{\text {th }}, 2011$; revised December $24^{\text {th }}, 2011$; accepted January $9^{\text {th }}, 2012$

\begin{abstract}
We found carbon nanotube (CNT) materials by the pyrolysis of the composite film of poly (vinyl alcohol) (PVA) reinforced with modified fly ash (FA) at $500^{\circ} \mathrm{C}$ for $10 \mathrm{~min}$ under $2 \mathrm{~L} / \mathrm{min}$ flow of nitrogen. Fly ash was treated with $2 \mathrm{M}$ sodium hydroxide and used with PVA to fabricate the composite film by aqua casting. CNT materials were analyzed using XPS, Raman, SEM and TEM. The admixtures of CNT materials and FA are a potential filler material for fabricating composites with polymer and metal. The process is an eco-friendly recycling paradigm for using value-added advanced products for the proper management of sustainable waste materials, plastic and FA.
\end{abstract}

Keywords: Poly (Vinly Alcohol); Modified Fly Ash; Composite and Carbon Nanotube

\section{Introduction}

Carbon nanostructured materials were synthesized by pyrolysis of benzene at $1000^{\circ} \mathrm{C}$ over Ni powder for 60 min [1]. Carbon nanocapsules with silicon carbide nanoparticles were reported by thermal decomposition of PVA at $500^{\circ} \mathrm{C}$ in argon [2]. Catalytic graphitization was reported using PVA with an iron-containing catalyst for the formation of CNTs at $600^{\circ} \mathrm{C}-800^{\circ} \mathrm{C}$ in nitrogen flow for $2 \mathrm{~h}$. The catalyst particles were uniformly distributed on the surface and filled the hollow channel of CNTs. The CNT structures depended on the nature of the catalyst and carbon sources as well as the pyrolysis conditions [3].

Coal combustion in power stations generates huge amounts of FA as a by-product. The storage and proper disposal of this material are a significant global concern on both environmental and economic fronts [4]. FA is commonly processed by disposal as landfill in dams and lagoons. It typically consists of crystalline aluminosilicate, mullite and $\alpha$-quartz ( $\sim 96.5 \%)$ spherical particles between $1 \mu \mathrm{m} \sim 100 \mu \mathrm{m}$ in diameter, along with traces of metal oxides $(\sim 3.5 \%)$ (iron, calcium, magnesium, potassium, sodium, titanium), depending on the processing conndition and the chemical composition of the coal burned [5].

In 2007 , less than $20 \%$ of 14.5 million tons produced in Australia was re-used [6]. The remaining FA was dis-

${ }^{*}$ Corresponding author. posed in low land area. Successful results have been achieved in the use of FA of different researchers are in agriculture and soil management, the production of polymer composites [5] cement and concrete [7], metal composites [8] as catalytic support for hydrogen production, steam methane reforming [4] and iron-nitrate-impregnated support for the synthesis of multi-walled CNTs (MWCNTs) [6].

Research on the recycling of FA as a filler and catalyst support has been moving towards a green technology for value-added products and composite materials. We have recently reported the dual functions of fly ash as filler and catalyst for the synthesis of CNT materials [9]. We report here a significantly more cost-effective pyrolysis method than the expensive arc-discharge and CVD methods, using waste composite film of PVA and modified FA for growth and self-assembled CNT ropes, where FA has dual roles as filler in composite film and catalyst in pyrolysis condition.

\section{Experimental Procedures}

\subsection{Materials}

An industrial waste FA material was obtained from Swanbank Coal Fire Plant, Qld, Australia. The PVA (molecular weight, $125,000 \mathrm{~g} / \mathrm{mol}$; degree of hydrolysis $\sim 89 \%$ with remaining $11 \%$ acetate) from S.D. Fine-Chemical Ltd., Chennai, India, and reagent grade sodium hydroxide from Laboratory Unilab Reagent, Sydney, were purchased and used as received. 


\subsection{Preparation of Composite Films}

The detailed modification process of FA with sodium hydroxide at $85^{\circ} \mathrm{C}$ for $8 \mathrm{~h}$ was reported in our earlier research. The PVA composite film was fabricated by a casting method from an aqueous suspension of PVA and modified FA. The PVA was dissolved in distilled water at $80^{\circ} \mathrm{C}$ for $2 \mathrm{~h}$ to prepare the $1.2 \mathrm{wt} \%$ solution. The modified FA particles $(5 \mathrm{wt} \%)$ were dispersed and sonicated for $5 \mathrm{~min}$. The resulting mixture was cast in glass petri dishes (100 $\mathrm{mm}$ diameter) and bubbles were removed by shaking and air blowing. The petri dishes were kept at room temperature until completely dry. The films were peeled out and dried in an oven at $60^{\circ} \mathrm{C}$ under vacuum for $6 \mathrm{~h}$. The thickness of the films was $50 \mu \mathrm{m}-70 \mu \mathrm{m}$ [5].

\subsection{Pyrolysis Method}

The pyrolysis of the samples was run at an isothermal condition in a horizontal quartz tube furnace (HTF 6035) under nitrogen flow. The hot zone of the furnace was preheated to $500^{\circ} \mathrm{C}$ using thermocouple. The furnace connection scheme was as reported in our work [9]. The weighed amount of sample was kept in an alumina crucible and placed on a graphite feeder, which was inserted into the cold zone with simultaneous nitrogen purging at $2 \mathrm{~L} / \mathrm{min}$, held for $30 \mathrm{~s}$, and passed through into the hot zone for $10 \mathrm{~min}$. After pyrolysis, the sample was relocated from hot to cold zones via the graphite feeder for $30 \mathrm{~s}$ and removed from the alumina crucible at room temperature. The sample was stored in a small bottle, and used for calculation and analysis.

\subsection{Analysis and Analytical Instruments}

X-ray photoelectron spectroscopy (XPS) was conducted using an ESCALAB220i-XL (VG Scientific, Thermo Scientific, UK) to calculate the atomic composition of the CNTs. The standard conditions were used: $>2 \times 10^{-9}$ mbar, mono-chromated Al K alpha (energy $1486.6 \mathrm{eV}$ ), $200 \mathrm{~W}(20 \mathrm{~mA}$ and $10 \mathrm{kV})$, spot size $\sim 1 \mathrm{~mm}$ in cross, photoelectron take-off angle of 90, pass energy of 100 $\mathrm{eV}$ for survey scans and $20 \mathrm{eV}$ for region scans, and step-sizes of $1 \mathrm{eV}$ for survey scans and $0.1 \mathrm{eV}$ for region scans.

Raman spectroscopy was performed on an inVia Reinshaw Raman microscope using green laser excitation $(514 \mathrm{~nm})$ for the calculation of the degree of ordered CNT structures. Scan was recorded on an extended range $\left(100 \mathrm{~cm}^{-1}-4000 \mathrm{~cm}^{-1}\right)$, with $10 \%$ power of $1.2 \mathrm{~mW}$. The samples were prepared by pressing onto a glass slide.

A Hitachi 4500-II scanning electron microscope (SEM) was used to examine the morphology of the sample. The sample on carbon tape was coated with chromium in a chromium sputter unit to improve conductivity. Transmi- ssion electron microscopy (TEM) images were obtained using a Philips CM200 with $200 \mathrm{kV}$. The sample was dispersed in ethanol and a drop of diluted suspension was poured onto a carbon-coated copper grid, which was directly injected into a sample injection holder after airdrying for three days.

\section{Results and Discussion}

The pyrolysis of PVA composite film with $5 \mathrm{wt} \%$ modified FA left behind black solid residue on the alumina crucible. The yield was $53.8 \%$ depending on the FA contents in the composite films.

$\mathrm{X}$-ray photoelectron spectroscopy (XPS) was conducted using an ESCALAB220i-XL to calculate the atomic carbon composition in the solid residue [10]. XPS spectrum of the material is shown in Figure 1. The peaks at $285.1 \mathrm{eV}$ and $532.2 \mathrm{eV}$ were generated by photoelectrons emitted from the carbon $1 \mathrm{~s}$ and oxygen $1 \mathrm{~s}$ core levels, respectively [10].

The broad peak was isolated by the Lorentzian method to divide and fit the curve by composed of five peaks [9]. The peak at $285.1 \mathrm{eV}$ was generated by the excitation of $\mathrm{C}-\mathrm{C} / \mathrm{C}-\mathrm{H}$ bonds in the graphite configuration with $\sim 83.8 \%$, including its shake-up structure. The peak was centred at $286.6 \mathrm{eV}$, which can be attributed to photoelectrons emitted from carbon atoms bonding with $\mathrm{C}-\mathrm{O}-\mathrm{H}$ group configuration with $6.8 \%$. Three other small peaks were centred at 288.1, 289.2 and $290.7 \mathrm{eV}$, emitted from the carbon atoms belonging to $\mathrm{C}-\mathrm{O}-\mathrm{C}(4.1 \%), \mathrm{C}=\mathrm{O}(3.6 \%)$ and $-\mathrm{COOH} /-\mathrm{COOCH}_{3}(1.6 \%)$ groups, respectively.

Therefore, it can be assumed that oxygen is a component of CNTs. The intensity of the oxygen $1 \mathrm{~s}$ core level

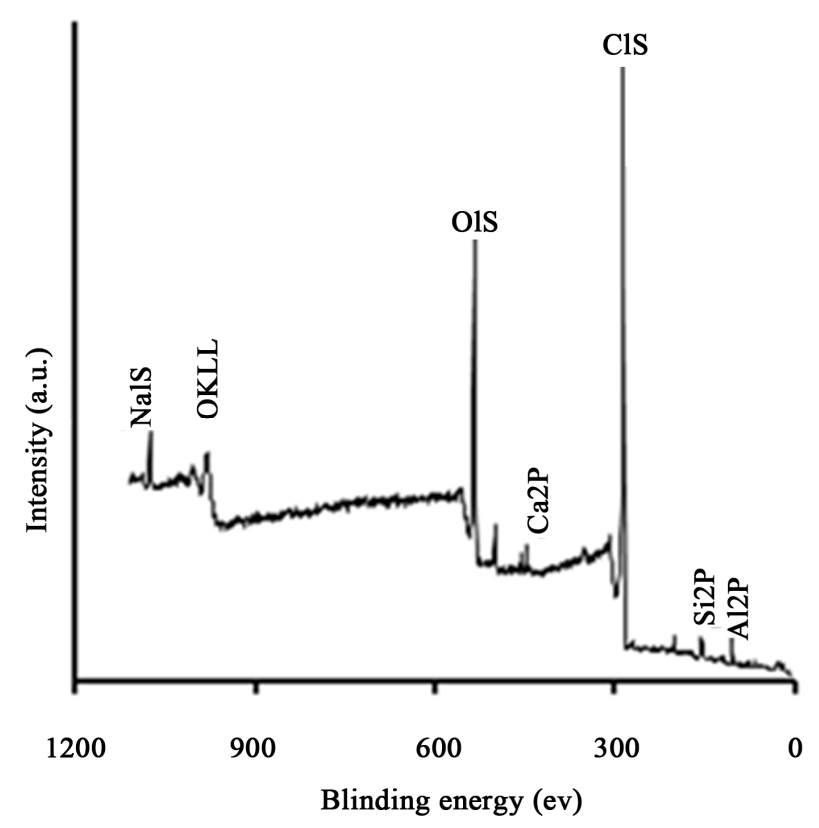

Figure 1. Wide-range XPS spectrum of CNT materials. 
was much higher than those of the Si and Al 2 s core levels. The relative concentration of oxygen and carbon are nearly $18 \%$ and $74 \%$, respectively; the remainder consisted of mixtures of $\mathrm{Na}, \mathrm{Si}, \mathrm{Ca}$ and $\mathrm{Al}$ in the CNT sample. However, the percentages of $\mathrm{O}, \mathrm{Si}$ and $\mathrm{Al}$ were $\sim 60.2 \%, 25 \%$ and $6 \%$, respectively, including $4 \%$ unburned carbon in FA, which was of amorphous structure.

The graphitic configurations of CNT materials were analyzed by Raman spectroscopy for more reliable authentication of the carbon structure. The characteristic peaks were identified and shown in Figure 2 on excitation with $514 \mathrm{~nm}$ laser light.

The strongest Raman $\mathrm{G}$ band line, the characteristic peak for well-graphitised CNT, appeared at $1598 \mathrm{~cm}^{-1}$ with relatively narrower FWHM, $71 \mathrm{~cm}^{-1}$. Another peak at $1380 \mathrm{~cm}^{-1}$ with $112 \mathrm{~cm}^{-1}$ FWHM is the D-line band, indicating a typical sign for defective graphitic structures on CNT. The intensity of the D-line is lower than that of the G-line, evidence of the more ordered carbon structure in CNT. CNT materials consist mainly of crystalline graphitic carbons along with amorphous carbons. The intensity of isolated peaks was calculated and used to determine the characteristic features of the graphitic wall of CNT materials [6].

The intensity ratio $(I \mathrm{G} / I \mathrm{D})$ of the $\mathrm{G}$ band and $\mathrm{D}$ band was treated to determine the degree of carbon graphitization in CNT configurations. The higher $I \mathrm{G} / I \mathrm{D}$ ratio indicated a greater degree of wall graphitization, suggesting superior CNT quality. The $I \mathrm{G} / I \mathrm{D}$ ratio of the CNT materials grown on FA surfaces was 3.43, higher than the 1.17 for MWCNTs yielded for iron-catalyst-coated FA by the CVD method [6].

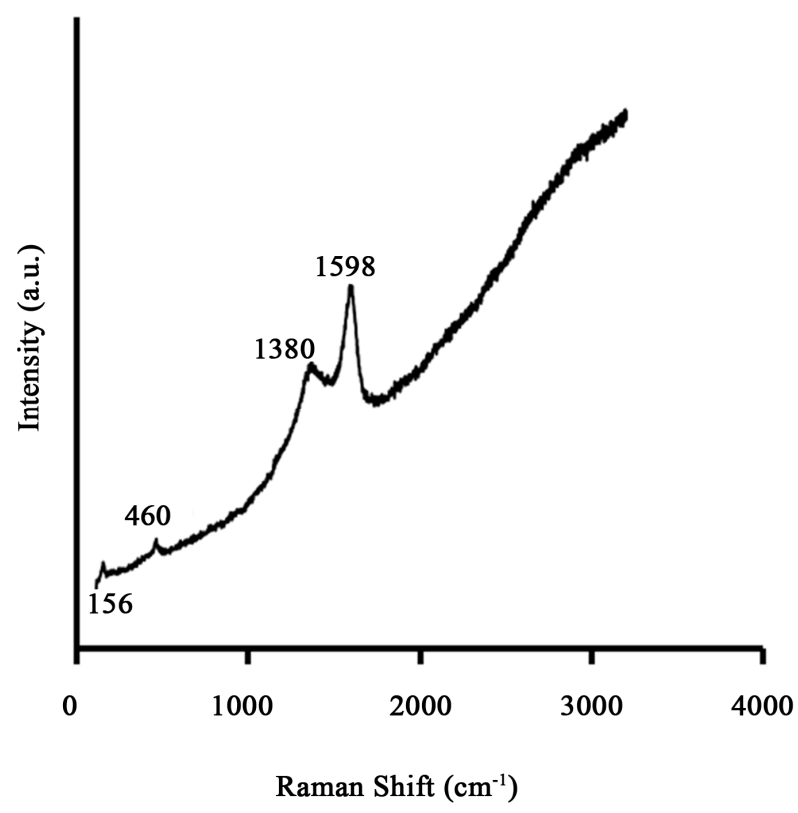

Figure 2. Raman spectrum of CNT.
The line width and intensity ratio of $(I \mathrm{G} / I \mathrm{D})$ varied depending on the structure of the carbons and the conditions used for analysis, e.g., laser power and energy [11]. The well-known phenomenon, Raman band intensities and widths, increased with the decrease of wavelength of exciting laser, whereas these lines were shifted to lower wave number regions with an increase of the exciting laser power $[12,13]$.

A Hitachi 4500-II scanning electron microscope (SEM) was used to examine the morphology of the sample. The morphology of the solid residue was visualized and analyzed by SEM and TEM. The morphologies of CNT ropes in SEM images are presented in Figures 3(a)-(b). Figure 3(a) clearly shows the active site where CNT ropes were initiated and grown randomly with curved and entangled structures on the FA surfaces.

Figure 3(b) shows several isolated, long, flexible CNT ropes captured with a $500 \mu \mathrm{m}$ scale bar. The ropes were observed in highly entangled with tightly twisted structures. The self-assembly of two, three and complicated

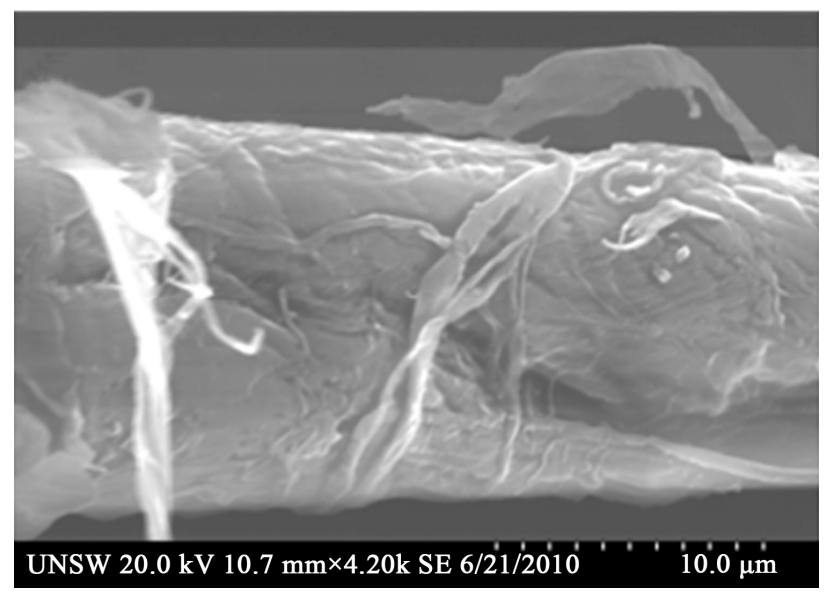

(a)

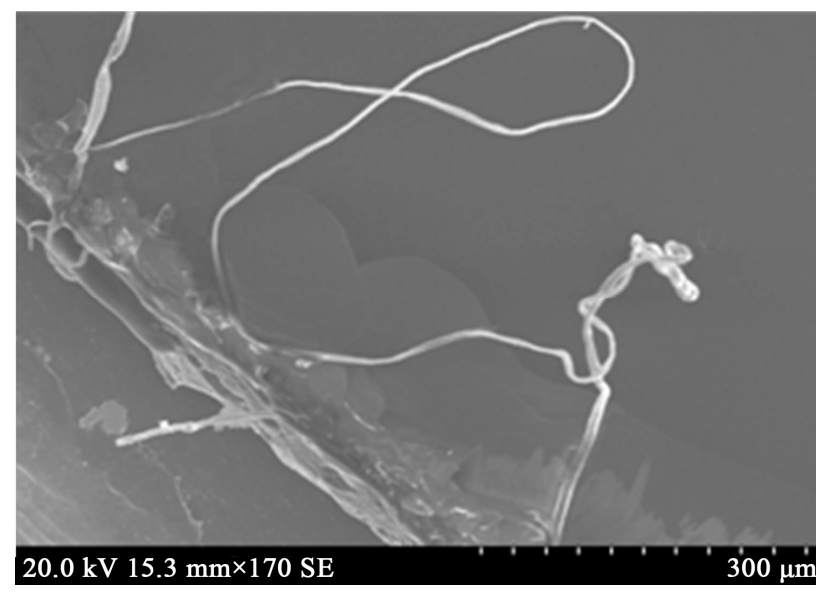

(b)

Figure 3. Representative SEM images of CNT ropes: (a) Random growth on active sites with curved and entangled geometry; (b) Isolated flexible ropes grown on FA surfaces. 
knitted CNT ropes also appeared in the multidirectional pathways. No effects of higher temperature, FA concentration and time on the growth and structure of CNT ropes were observed in the system. The surfaces of CNT ropes were not absolutely free from FA particles. There was FA particle adherence to some areas of the CNT ropes, suggesting 1) defects in graphitization permitted by the presence of space/void for FA particle deposition, and/or 2) the presence of functionalized carbon, which strongly interacts with FA [9].

We presented a simple and in-situ environmentally friendly method for self-assembled CNT ropes with indefinitely long and highly flexible permanent twisted microscopic structures in TEM images in Figures 4 (a)-(b). In term of growth mechanism, several models have been proposed by the decomposition of hydrocarbon on metal surfaces $[3,11]$, although the exact modes of action are still hypothetical.

The mechanism of CNT growth was studied with respect to carbon dissolution in a metal catalyst. The dissolved carbon produced by the decomposing of PVA at temperatures $600^{\circ} \mathrm{C}-800^{\circ} \mathrm{C}$ by an intermediate reaction on the catalyst surface, iron oxide. The dissolved carbon diffused through the metallic particle to form the layers of the growing CNT. For this reason, metallic particles were present at the ends of the CNT [3].

Considering the results obtained in the pyrolysis of PVA composite with unmodified FA, we have recently proposed a plausible overall growth mechanism for CNT structure [12]. A building block mechanism was considered with a broken TEM image of CNT to define a hypothetical model [14]. We observed the deposition of different sizes building blocks nearby on the broken edge of the tube in TEM image by exposure of sonication and high intense of electron beam. The different sizes of the broken building blocks were inferred from the results of different degrees of polymerization of PVA, since each of the PVA chains forms a building block in CNT structure [14]. We believe CNTs from the pyrolysis of PVA composite film with modified FA also formed followed by similar mechanism.

\section{Conclusion}

The growths of CNT on modified FA surfaces were visualized with different geometric self-assembly, e.g., bundles of CNT in ropes, twisted ropes, Y-branch ropes and staked-cone sheet. The mixture materials of self-assembled CNT ropes with modified FA as filler therefore point for large-scale application with polymer for potential fabricating composite materials. Another advantage of CNT materials is that the carbon chain is already partially functionalized, e.g., C-O-H (6.8\%), C-O-C (4.1\%), $\mathrm{C}=\mathrm{O}(3.6 \%)$ and $-\mathrm{COOH} /-\mathrm{COOCH}_{3}(1.6 \%)$, with pros-

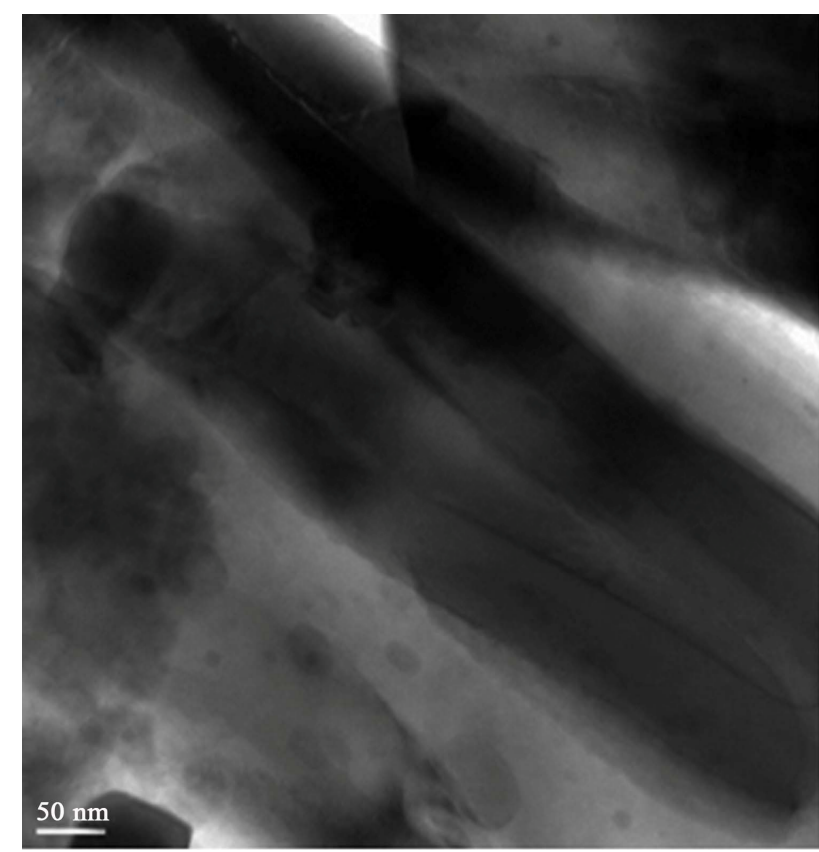

(a)

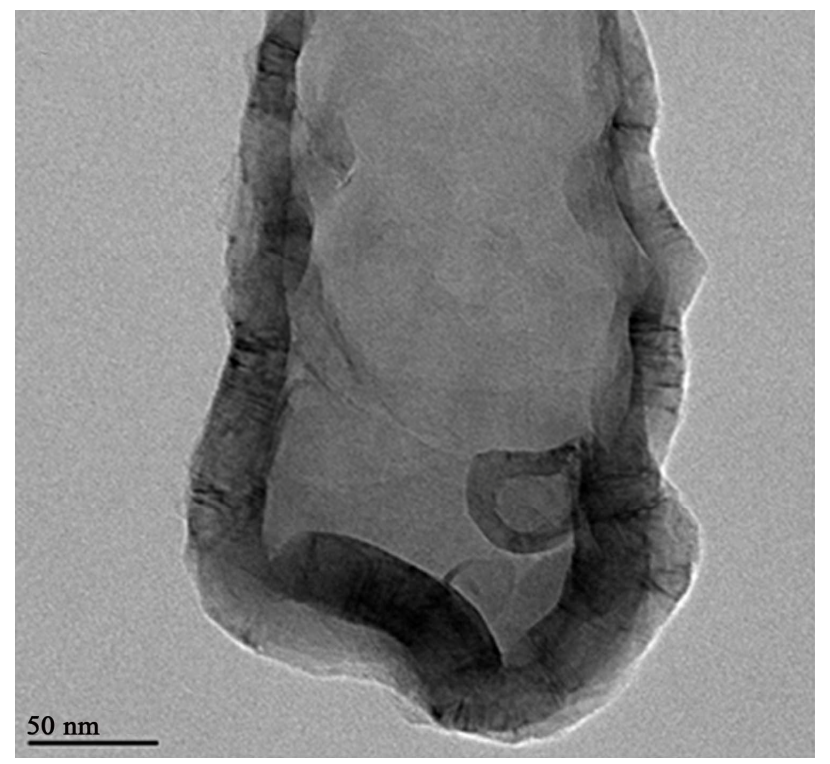

(b)

Figure 4. TEM images of higher magnification of a straight rope showing (a) CNT bundle and (b) flexible CNT rope.

pects for making hybrid bridges by covalent reactions with organic polymers for new classes of materials.

\section{REFERENCES}

[1] M. Benito, Y. Maniette, Y. Aniette, E. Mogoza and T. M. Martnez, "Carbon Nanotubes Production by Catalytic Pyrolysis of Benzene," Carbon, Vol. 36, No. 5-6, 1998, pp. 681-683. doi:10.1016/S0008-6223(98)00039-6

[2] T. Oku, K. Niihara and K. Suganuma, "Formation of Carbon Nanocapsules with SiC Nanoparticles Prepared 
by Polymer Pyrolysis," Journal of Materials Chemistry, Vol. 8, No. 6, 1998, pp. 1323-1325. doi: $10.1039 / \mathrm{a} 801912 \mathrm{f}$

[3] O. P. Krivoruchko, N. I. Maksimova, V. I. Zaikovskii and A. N. Salanov, "Study of Multiwalled Graphite Nanotubes and Filaments Formation from Carbonized Products of Polyvinyl Alcohol via Catalytic Graphitization at $600^{\circ} \mathrm{C}-800^{\circ} \mathrm{C}$ in Nitrogen Atmosphere," Carbon, Vol. 38, No. 7, 2000, pp. 1075-1082. doi:10.1016/S0008-6223(99)00225-0

[4] S. Wang, "Application of Solid Ash Based Catalysts in Heterogeneous Catalysis," Environmental Science and Technology, Vol. 42, No. 19, 2008, pp. 7055-7063. doi:10.1021/es $801312 \mathrm{~m}$

[5] D. C. D. Nath, S. Bandyopadhyay, A. Yu, D. Blackburn and C. White, "High Strength Bio Composite Films of Poly (Vinyl Alcohol) Reinforced with Chemically Modified-Fly Ash," Journal of Materials Science, Vol. 45, No. 5, 2010, pp. 1354-1360. doi:10.1007/s10853-009-4091-6

[6] O. M. Dunens, K. J. Mackenzie and A. T. Harris, "Synthesis of Multiwalled Carbon Nanotubes on Fly Ash Derived Catalysts," Environmental Science and Technology, Vol. 43, No. 20, 2009, pp. 7889-7894. doi:10.1021/es901779c

[7] J. P. Gorninski, D. C. D. Molin and C. S. Kazmierczak, "Study of the Modulus of Elasticity of Polymer Concrete Compounds and Comparative Assessment of Polymer Concrete and Portland Cement Concrete," Cement \& Concrete Research, Vol. 34, No. 11, 2004, pp. 2091-2095. doi:10.1016/j.cemconres.2004.03.012

[8] P. K. Rohatgi, N. Gupta and S. Alaraj, "Thermal Expan- sion of Aluminum-Fly Ash Cenosphere Composites Synthesized by Pressure Infiltration Technique," Journal of Composite Materials, Vol. 40, No. 13, 2006, pp. 11631173. doi: $10.1177 / 0021998305057379$

[9] D. C. D. Nath and V. Sahajwalla, "Application of Fly Ash as a Catalyst for Synthesis of Carbon Nanotube Ribbons," Journal of Hazardous Materials, Vol. 192, No. 2, 2011, pp. 691-697. doi:10.1016/j.jhazmat.2011.05.072

[10] J. E. Moulder, W. F. Stickle, P. E. Sobol and K. D. Bomben, "Handbook of X-Ray Photoelectron Spectroscopy," Perkin Elmer Corporation, Waltham, 1992.

[11] A. A. Didik, V. I. Kodolov, A. Y. Volkov, E. G. Volkova and K. H. Hallmeier, "Low-Temperature Growth of Carbon Nanotubes," Inorgnic Materials, Vol. 39, No. 6, 2003, pp. 583-587. doi:10.1023/A:1024045002419

[12] J. Kastner, T. Pichler, H. Kuzmany, S. Curran, W. Blau, D. N. Weldon, M. Delamesiere, S. Draper and H. Zandbergen, "Resonance Raman and Infrared Spectroscopy of Carbon Nanotubes," Chemistry and Physics Letter, Vol. 221, No. 1-2, 1994, pp. 53-58. doi:10.1016/0009-2614(94)87015-2

[13] Y. Ando, X. Zhao and H. Shimoyama, "Structure Analysis of Purified Multiwalled Carbon Nanotubes," Carbon, Vol. 39, No. 4, 2001, pp. 569-574. doi:10.1016/S0008-6223(00)00162-7

[14] D. C. D. Nath and V. Sahajwalla, "Growth Mechanism of Carbon Nanotube Produced by Pyrolysis of a Composite Film of Poly (Vinyl Alcohol) and Fly Ash," Applied Physics A, Vol. 104, No. 2, 2011, pp. 539-544. doi:10.1007/s00339-011-6405-1 